\title{
PREPARATION AND ANALYSIS OF PCL SPUN CHITOSAN SCAFFOLDS AS GUIDANCE CHANNELS FOR PERIPHERAL NERVE REGENERATION
}

\author{
Dipl.-Ing. M.Sc. Peter Behrens ${ }^{1}$, Prof. Dr.-Ing. Birgit Glasmacher ${ }^{1}$, \\ Sven Duda ${ }^{2}$, Prof. Dr. med. vet. Kirsten Haastert - Talini² \\ ${ }^{1}$ Institute for Multiphase Processes (IMP), Leibniz Universität Hannover, Germany \\ ${ }^{2}$ Institute of Neuroanatomy, Hannover Medical School, Germany
}

Behrens.Proehl@t-online.de

\begin{abstract}
The results of this work show that the process of oriented solidification and lyophilisation is able to produce porous chitosan scaffolds with appropriate porosity and pore size for nerve regeneration. Interesting in this context are the results of statistical analysis of image analysis from SEM micrographs of uncrosslinked and $U V$ cross-linked samples. The average pore size and mean minimum pore diameter show only small differences if the cooling rate is varied from $B=1 . .5 \mathrm{~K} / \mathrm{min}$ and the temperature gradient from $G=1,1.5,2.0 \mathrm{~K} / \mathrm{mm}$. The average pore size (cross sectional area) of these samples can be estimated with reasonable accuracy, with 2100 $\mu \mathrm{m}^{2}$. The average minimum pore diameter is within the range of 36-38 $\mu \mathrm{m}$. These values are in a favourable range for the cell growth of nerve regeneration.
\end{abstract}

Keywords: nerve guidance channel, oriented solidification, lyophilisation, chitosan, electrospinning of PCL

\section{Introduction}

This work deals with the preparation and analysis of PCL spun chitosan scaffolds as guidance channels for peripheral nerve regeneration. It represents the cellular and neuroanatomical basics of the regeneration of peripheral nerves, and the current medical state of the treatment of injuries of the peripheral nervous system. The biological process of peripheral nerve regeneration is considered in detail and based on that the requirements of a functionally active nerve guidance channel (NGC) are described. It is being studied what materials for the design of a nerve guidance channel are in principle promising. Favourable materials are especially biologically degradable materials. Therefore there are nerve guidance channels produced from the biodegradable materials chitosan and polycaprolactone PCL. These nerve guidance channels are examined more closely. The properties of chitosan and PCL and the state of science in the use of these materials as nerve guidance channels are presented.

\section{Methods}

The nerve guidance channels in these studies are produced by a multistage manufacturing process. A porous scaffold structure of chitosan is generated by oriented solidification and lyophilisation. In a next step, the chitosan scaffold is weaving around by electrospinning with PCL fibers. The chitosan scaffolds can be treated by various strategies for crosslinking, with the aim to achieve a higher structural stability. It is analysed that a UV crosslinking of the chitosan as well as the crosslinking with Gluteralaldehyd affects the pore structure of the chitosan scaffolds. In the preparation of the samples the freezing parameters, cooling rate $\mathrm{B}(\mathrm{B}=1 \ldots 5 \mathrm{~K} / \mathrm{min})$ and temperature gradient $\mathrm{G}(\mathrm{G}=1,1.5,2.0 \mathrm{~N} / \mathrm{mm})$ are varied. From the samples scanning electron microscopic recordings (see Figure 1 (a)) are created and evaluated by using image analysis methods for further investigation. The results of image analysis are then statistically analyzed with the aim to determine statistical parameters related to the average pore size and the minimum pore diameter. The distribution of the average pore size and the mean minimum pore diameter are compared by means of statistical tests. Further studies on the improvement of producing an electrospun PCL cover for the nerve guidance channels are carried out with a rotator (see figure 1 (b)) which has been developed at the Institute for Multiphase Processes. Investigations at defined process parameters determine the optimum distance of the rotary device and the fibre diameter as a function of distance.

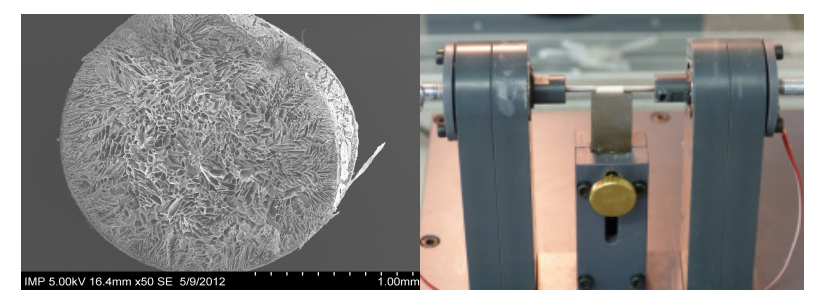

Figure 1: (a) SEM micrograph of a chitosan scaffold and (b) Rotation device for electrospinning the samples with PCL fibres

The nerve guidance channel is surgically attached to the peripheral nerves. To examine whether the compound is sufficiently stable tensile tests are carried out with nerve guidance channels fixed at rat nerve. 


\section{Results}

The average pore size (cross sectional area) of all examined uncrosslinked samples for the freezing parameter B $=1.5 \mathrm{~K} / \mathrm{min}$ and $\mathrm{G}=1,1.5,2 \mathrm{~N} / \mathrm{mm}$ is in accordance with the statistical evaluation system $2101 \mu \mathrm{m}^{2}$. The median is $2076 \mu \mathrm{m}^{2}$. For the UV crosslinked samples the average value of the mean pore size of $2121 \mu \mathrm{m}^{2}$ and the median $2090 \mu \mathrm{m}^{2}$. The average minimum pore diameter of the uncrosslinked chitosan samples is $38.6 \mu \mathrm{m}$, that of the UV crosslinked samples $36.5 \mu \mathrm{m}$. The calculated mean pore sizes and average minimum pore diameters are to be regarded as advantageous for the regeneration of peripheral nerve cells. The investigations of the pore morphology of the uncrosslinked or UV crosslinked samples indicate that the samples have in the mean pore size statistically no significant differences. However, there are significant differences of the average minimum pore diameter for both distributions. This indicates a change in the shape of the pores between the uncross-linked and UV crosslinked chitosan samples. This is also visible in the SEM images. The results of the cross-linking with Gluteralaldehyd show a more pronounced influence on the pore structure and morphology of the scaffolds. This is regarded as unfavourable.

It can be shown that the favourable spinning distances from the rotation device are within the range of 22.5 to $25 \mathrm{~cm}$. A voltage of $24.3 \mathrm{kV}$ and $27 \mathrm{kV}$ is set for the formation of a stable Taylor cone for the process of electrospinning. At a flow rate of $0.2 \mathrm{ml} / \mathrm{h}$ thus can provide stable process conditions.

The results of the tensile tests show that the nerve guidance channel itself is sufficiently stable and the system fails in the transition between the nerve tissue and suture through tearing.

\section{Discussion}

The results of this work show that it is possible to produce by means of oriented solidification and lyophilisation chitosan scaffolds with a characteristic pore size and pore morphology. The results of the analysis of the pore morphology of the chitosan scaffolds show that the average pore size and the mean minimum pore diameters are in an order of magnitude that offers favourable conditions for enabling regeneration of peripheral nerves. The internal geometry of the scaffold should be designed suitable for acting as a guidance matrix for the axonal regeneration and not acting as a physical barrier. In one study [1] it is determined that a tube diameter of the micro channels of 20-30 $\mu \mathrm{m}$ is a particularly favourable geometric condition for leading the growth of neurons. The range for the micro channel size should be between $20-60 \mu \mathrm{m}$. These magnitudes match very well with the determined pore sizes and mean minimum pore diameter in this investigation.

Regarding to the general need for the addition of crosslinking chemicals to the chitosan scaffolds of nerve guidance channels it should be noted that the results of tests using nerve guidance channels that have been produced at the Institute for Multiphase Processes at Leibniz Univer- sity of Hannover show in in-vivo rat tests at the Institute of Neuroanatomy at the Hannover Medical School, that also without a cross-linking these chitosan scaffolds are mechanically and chemically stable and support the regeneration of peripheral nerves. The preliminary results of the in-vivo tests suggest that even without neutralisation and without crosslinking the chitosan scaffolds enable favourable conditions for regeneration of peripheral nerves. The scaffolds are of sufficient spatial and temporal stability to act as temporary nerve guidance channels. It seems that the chitosan scaffold does not act as a physical barrier, which prevents regeneration. It can therefore be assumed that chitosan provide a basically good suitability as a potential material for use as a nerve guidance channel. Regarding the suitability of porous chitosan scaffolds it is found in tests [2] that the permeability is better suitable than PLGA for use as nerve guidance channels. It can also be shown that porous structures, which are produced by oriented solidification, support the ingrowths of cells [3]. The results show that overall, the use of porous chitosan scaffolds generated by oriented solidification and lyophilisation [4] is a very promising way for the future development of a nerve guidance channel.

\section{Acknowledgement}

For academic supervision of this work and the stimulating discussions I thank Prof. Dr. Ing Birgit Glasmacher, Dipl.-Ing. Soenke Wienecke, Mr. Tanmay Chakradeo, Ph.D. M.Sc. and Dipl.-Ing. Tarik Attia from the Institute for Multiphase Processes at the Leibniz University of Hannover, Germany and Prof. Dr. med. vet. Kirsten Haastert - Talini and Mr. Sven Duda from the Institute of Neuroanatomy, Hannover Medical School.

\section{Bibliography}

[1] Mahoney, M.J., Chen, R.R., Tan, J., Saltzman, W.M.: The influence of microchannels on neurite growth and architecture, Biomaterials 26 (7), 771-778, 2005.

[2] Huang, Y., Huang, Y.: Tissue engineering for nerve repair in Biomedical Engineering-Applications; Basis \& Communications, 100-110, 2006.

[3] Deumers, R., Bozkurt, A., Meek, F.M., Marco, M.A.E., Joosten, E.A.J., Weis, J., Brook, G.A. : Repairing injured peripheral nerves: bridging the gap, Progress in Neurobiology 92, 245-276, 2010.

[4] Glasmacher, B., Wienecke, S., Chakradeo, T., Duda, S., Haastert - Talini, K.: Nerve guides using a three- dimensionally structured chitosan core with an electrospunn poly-caprolactone shell for peripheral nerve reconstruction, Journal of Tissue Engineering and Regenerative Medicine, Volume 6, issue supplement s1, 75, 2012. 